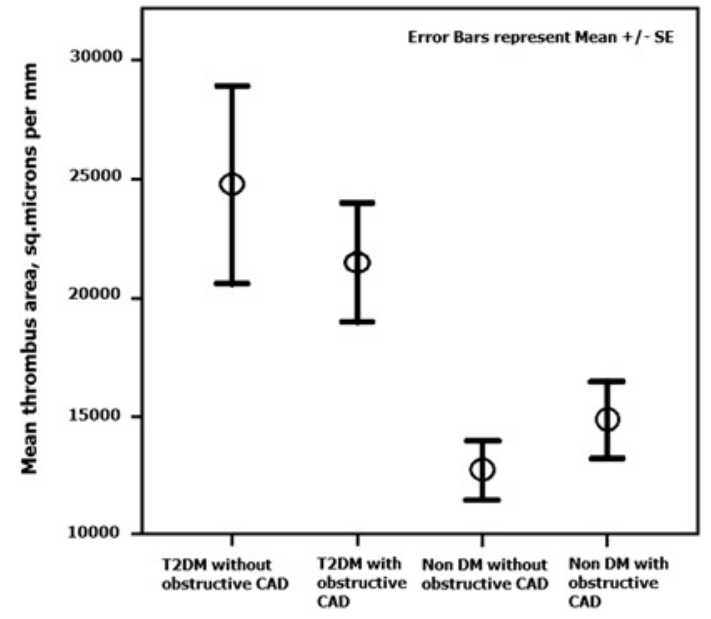

Abstract 113 Figure 1 Oneway ANOVA showed significant difference bettween the groups, $F=5.26, p=0.002$. df (3.76). Post hoc compansion with Bonferroni test showed that BT was significantly higher in T2DM with non-obstructive CAD compared to the other three groups $p<0.05$.

angiography through an independent investigator blinded to clinical and laboratory data. Lesions were classified as either obstructive (plaque $>75 \%$ of the luminal diameter) or non-obstructive (plaque $25 \%-75 \%$ of luminal diameter). We excluded those with minor plaques ( $<25 \%$ of the luminal diameter) and systemic inflammation (eg, sepsis). The subjects were divided: (i) T2DM and obstructive $\mathrm{CAD}(\mathrm{n}=26)$, (ii) T2DM and non-obstructive CAD ( $\mathrm{n}=14$ ), (iii) nondiabetic and obstructive $\mathrm{CAD}(\mathrm{n}=30)$ and (iv) non-diabetic and nonobstructive CAD $(n=10)$. BT was measured by the ex-vivo Badimon chamber as total thrombus area. Cytokines and platelet activation markers were measured.

Results All baseline cardiovascular risk factors were similar between groups ( $p>0.05)$. BT was higher in T2DM than in those without (22 481 \pm 12336 vs $14650 \pm 8074, p=0.024)$. When stratified according to CAD status, BT was highest in those with T2DM and non-obstructive $\operatorname{CAD}(\mathrm{p}=0.002$, ANOVA $\mathrm{F}=5.26$ ) (Abstract 113 figure 1). Inflammatory cytokines TNF $\alpha \quad p=0.018$, ANOVA $\mathrm{F}=3.15)$ and interleukin $6(\mathrm{p}=0.031$, ANOVA $\mathrm{F}=5.0)$ and platelet

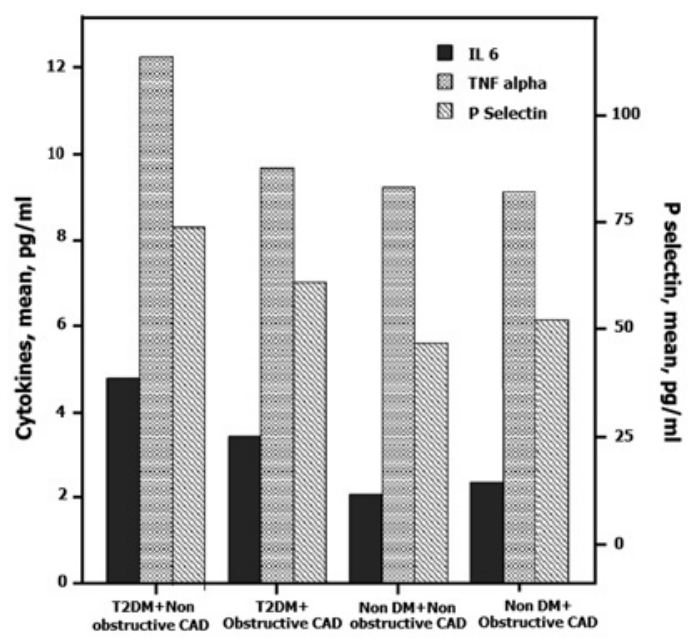

Abstract 113 Figure 2 Patients are grouped according to their diabetic status and coronary lesion characteristics. One way ANOVA for IL-6 $\mathrm{F}=5.006, p=0.03$, TNF $\alpha \mathrm{F}=3.153, \mathrm{p}=0.03$, P-selectin $\mathrm{F}=3.44, \mathrm{p}=0.02$, post hoc tests including Bonferroni showed t $p<0.05$ for multiple comparisions between T2DM+Non obstructive CAD group and the rest. activation as measured by $\mathrm{P}$ selectin were highest in this group. $(p=0.022$, ANOVA $F=3.422)$ (Abstract 113 figure 2). Interleukin 1, interferon $\gamma$ and soluble CD40 ligand levels were similar between the groups.

Conclusion Patients with T2DM and non-obstructive CAD had highest BT and markers of platelet activation and inflammation. These findings suggest biochemical changes also play a significant role in evolution of NSTE-ACS in T2DM. A causal link, if confirmed by large-scale studies may offer us an opportunity to identify therapeutic targets like individualised anti thrombotic therapy and anti inflammatory therapy in this high risk population.

\section{WHOLE GENOME SEQUENCING TO IDENTIFY GENETIC VARIANTS UNDERLYING CARDIOVASCULAR DISEASE AMONG INDIAN ASIANS}

doi:10.1136/heartjnl-2012-301877b.114

J C Chambers, ${ }^{*}$ S T Tan, W H Zhang, J Sehmi, A Al-Hussaini, M Ramasamy, J Scott, P Elliott, J S Kooner. Imperial College London, London, UK

Introduction Indian Asians have a twofold higher risk of cardiovascular disease compared to Europeans which is not explained by conventional cardiovascular risk factors or known genetic variants. The genetic architecture of Indian Asians has not previously been described. We hypothesised that whole genome sequencing of Indian Asians may identify both common and rare variants specific to this population that contribute to their increased cardiovascular disease risk.

Methods We carried out whole genome sequencing (mean depth $28.4 \times$ ) in eight men of Indian Asian origin participating in the London Life Sciences Population (LOLIPOP) study. Sequencing was carried out using paired end and mate pair libraries on an Illumina GA2 machine. Read alignment was done by BWA, and variants called using GATK and SAMtools. Sensitivity for single nucleotide polymorphism (SNP) detection was assessed by comparison to whole genome data.

Results We identified 6602840 autosomal variants, 436823 of which are novel SNPs. Of these, 50585 appear to be common (present at least twice, corresponding to minor allele frequency $>10 \%$ ). We found 21659 autosomal SNPs that were expected to affect protein coding, of which 2174 are novel. Among the coding SNPs identified, 145 are in genes linked to human diseases, such as obesity (FTO, UCP1), diabetes mellitus (CDKAL1, GCGR, HNF1B), lipid metabolism (APOB), hypertension (NOS2), and renal disease (NPHP4, PKD1). We also found 65613 novel autosomal indels of which 35097 are present at least twice, and 2301 novel deletions $>100 \mathrm{bp}$. We show that $>50 \%$ of the novel genetic variants are not in high LD ( $\left.22_{i} \mathrm{Y} 0.8\right)$ with tag SNPs and hence not captured on available high-density microarrays.

Conclusions We identify more than 500000 genetic variants not previously reported in 1000 genomes or dbSNP, and likely to be Indian Asian specific. The novel variants identified here are strong candidates for genetic factors underlying the increased risk of diabetes and cardiovascular disease among Indian Asians.

\section{UNIQUE CHARACTERISTICS OF CD14++CD16+ MONOCYTES IN PATIENTS WITH ACUTE HEART FAILURE AND IMPLICATIONS FOR CLINICAL OUTCOME}

doi:10.1136/heartjnl-2012-301877b.115

B J Wrigley, ${ }^{*}$ E Shantsila, L Tapp, G Y H Lip. University of Birmingham Centre for Cardiovascular Sciences, Birmingham, UK

Background Monocytes play important roles in inflammation, angiogenesis and tissue repair and may contribute to the 
Abstract 115 Table 1 Monocyte subset numbers

\begin{tabular}{llllll}
\hline & AHF & SHF & CAD & HC & p Value \\
\hline $\begin{array}{l}\text { Total } \\
\text { monocytes } \\
\text { (per } \mu \text { l) }\end{array}$ & $852 \pm 300 \mathrm{ABC}$ & $646 \pm 172 \mathrm{D}$ & $541 \pm 139$ & $502 \pm 190$ & $<0.001$ \\
$\begin{array}{l}\text { Mon1 } \\
\text { (per } \mu \text { l) }\end{array}$ & $685 \pm 224 \mathrm{ABC}$ & $524 \pm 156 \mathrm{D}$ & $448 \pm 120$ & $412 \pm 166$ & $<0.001$ \\
$\begin{array}{l}\text { Mon2 } \\
\text { (per } \mu \text { l) }\end{array}$ & 59.9 & 42.7 & 29.5 & 33.7 & 0.006 \\
$\begin{array}{l}\text { Mon3 } \\
\text { (per } \mu \text { l) }\end{array}$ & $78.0 \pm 54.5 \mathrm{C}$ & $71.2 \pm 33.0$ & $60.1 \pm 28.0$ & $55.2 \pm 29.4$ & 0.024 \\
\hline
\end{tabular}

$A=p<0.05$ AHF vs $S H F, B=p<0.05$ AHF vs $C A D, C=p<0.05$ AHF vs $H C, D=p<0.05$ SHF vs $\mathrm{HC}, \mathrm{E}=\mathrm{p}<0.05$ SHF vs $\mathrm{CAD}$.

pathophysiology of heart failure (HF). We examined differences in monocyte subset numbers and expression of cell surface markers of activation (CD14) and chemotaxis (CCR2) in patients with acute HF (AHF), stable HF (SHF) and controls and evaluated their impact on clinical outcomes.

Methods Three monocyte subsets [CD14++CD16-CCR2+ (Mon1), CD14++CD16+CCR2+ (Mon2) and CD14+CD16+ +CCR2- (Mon3)] were analysed by flow cytometry in 51 patients with AHF, 42 patients with SHF, 44 patients with stable coronary artery disease (CAD) and 40 healthy controls (HC). Surface marker expression of CD14 and CCR2 was also measured and expressed as median fluorescent intensity (MFI). The prognostic impact of monocyte subsets was examined in patients with AHF.

Results Patients with AHF had significantly higher Mon1 counts compared to other groups ( $\mathrm{p}<0.001$ for all) (Abstract 115 table 1 ). Similarly, Mon2 counts were increased in AHF compared to SHF $(p=0.011), C A D(p<0.001)$ and $\mathrm{HC}(p<0.001)$. Mon2 counts were also increased in SHF compared to both $\mathrm{CAD}$ and $\mathrm{HC}$ groups $(p=0.023, p=0.035$ respectively). In AHF, CD14 expression by Mon2 was significantly higher than in CAD patients (1481 473 vs $1228 \pm 408, p=0.039$ ) and in SHF patients, CD14 expression by Mon2 was significantly higher than in CAD patients (1502 \pm 484 vs $1228 \pm 408, \mathrm{p}=0.031)$. CCR 2 expression by Mon2 in AHF was higher than in HC (128 \pm 43.9 vs $104 \pm 28.5, p=0.013)$ and CCR2 expression by Mon2 was higher in SHF compared to HC (126士36.2 vs

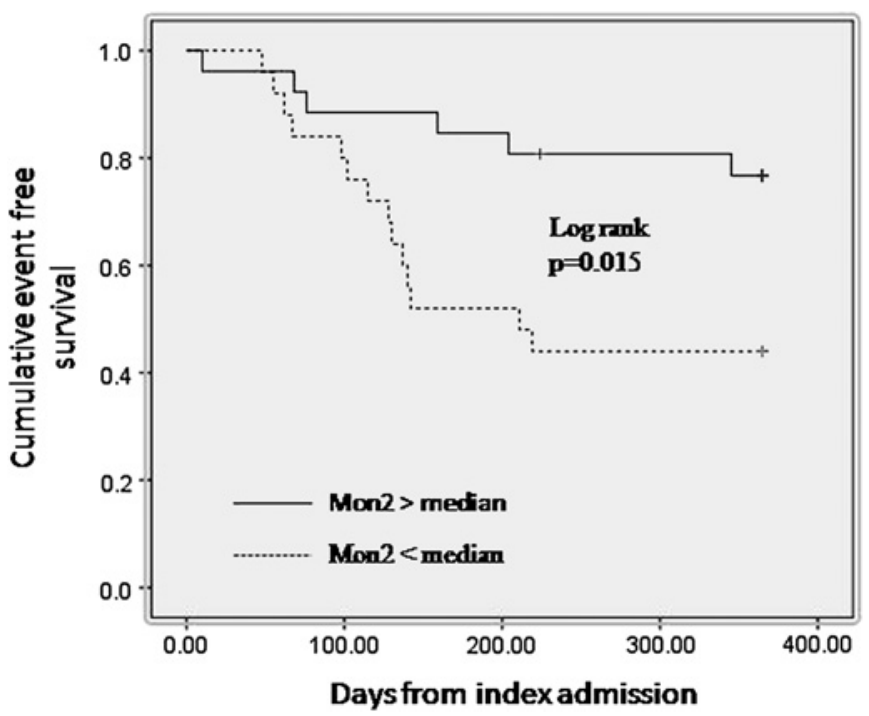

Abstract 115 Figure 1 Kaplan-Meier curves of cumulative event-free survival in patients with AHF for the primary end-point of mortality or rehospitalisation. The groups are divided along the median value of Mon2 counts $(59.9 \mathrm{cells} / \mu \mathrm{l})$.
104 $\pm 28.5, p=0.032) .20$ patients (39.2\%) with AHF reached the primary end point of death or re-hospitalisation, with a median time to event of 129 (IOR 70.0-209) days. In Cox regression analysis, after adjustment for age, left ventricular ejection fraction, serum creatinine and brain natriuretic peptide, Mon2 count remained an independent negative predictor of combined death and re-hospitalisation [HR (for an increase of 10 cells/ $\mu \mathrm{l}$ ) 0.829 (CI 0.713 to 0.964 ; $\mathrm{p}=0.015)]$. In Kaplan-Meier analysis AHF patients with Mon2 above median $(59.9$ cells/ $\mu \mathrm{l})$ had significantly better outcomes compared to those below the median (Abstract 115 figure 1).

Conclusion We have shown for the first time that CD14++CD16+ monocytes (Mon2) are an independent negative predictor of adverse prognosis in patients with AHF. This subset is phenotypically different from the other monocyte subsets, with increased expression of markers of activation (CD14) and chemotaxis (CCR2). Consequently, the Mon2 subset merits further evaluation as a prognostic marker and potential therapeutic target in patients with HF.

\section{MATRIX METALLOPROTEINASE INHIBITION ATTENUATES REPERFUSION INJURY, INDEPENDENTLY OF AND ADDITIVE TO MITOCHONDRIAL PERMEABILITY TRANSITION PORE INHIBITION}

doi:10.1136/heartjnl-2012-301877b.116

${ }^{1} \mathrm{R} \mathrm{M}$ Bell, ${ }^{*}{ }^{1}$ Cara Hendy, ${ }^{1} \mathrm{D}$ Bruce-Hickman, ${ }^{1} \mathrm{~S}$ Davidson, ${ }^{2} \mathrm{R}$ Breckenridge ${ }^{1} \mathrm{D}$ M Yellon. ${ }^{1}$ Hatter Cardiovascular Institute, Universtity College London, London, UK; ${ }^{2}$ MRC National Institute for Medical Research, London, UK

While matrix-metalloproteinase (MMP) inhibitors appear to protect against myocardial ischaemia/reperfusion injury, the mechanisms are poorly understood. We hypothesised that cardioprotection resultant from MMP inhibition is independent of mitochondrial permeability transition pore (mPTP), the end-effector of ischaemic postconditioning (iPOC). In ex-vivo and in-vivo mouse hearts, we investigated whether the MMP inhibitor, ilomostat $(0.25 \mu \mathrm{mol} / \mathrm{l})$, at the onset of reperfusion, could engender protection in the absence of cyclophillin-D (CyPD), an initiator of mPTP opening, against injurious ischaemia/reperfusion. We have previously demonstrated that CyPD knockout (KO) hearts have inherent resistance to ischaemia/ reperfusion injury, and cannot be further protected by either pre or post conditioning regimen. Therefore to further attenuate infarction in this model would require a mechanism independent of the mPTP. We found that ilomastat attenuated infarct size in wild type (WT) animals $(37 \% \pm 2.8 \%$ to $22 \% \pm 4.3 \%$, equivalent to ischaemic post conditioning (iPostC-6 cycles of $10 \mathrm{sec}$ reperfusion/ischaemia), $27 \% \pm 2.1 \%, \mathrm{p}<0.05)$. CyPD KO hearts had smaller infarcts compared to their conditioned WT brethren $(28 \% \pm 4.2 \%)$ and iPostC failed to protect, indicative of a pre-protected phenotype, yet ilomastat significantly attenuated infarct size in these hearts $(11 \% \pm$ $3.0 \%, p<0.001)$. Moreover, the infarct size seen in the ilomastat treated $\mathrm{KO}$ hearts was significantly smaller than that seen in the ilomastat treated WT hearts $(p<0.05)$, suggesting that mPTP and MMP inhibition is summative. Furthermore, ilomastat was found to be protective following prolonged injurious ischaemia (50 $\mathrm{min}$ ), an injurious regimen that iPostC in WT was insufficient to protect against $(49 \% \pm 7.8 \%$ to $31 \% \pm 2.8 \%, p<0.05)$. Interestingly, we found that delaying administration of ilomastat $15 \mathrm{~min}$ after the onset of reperfusion results in failure to protect the heart, indicating an effect upon early reperfusion injury, rather than post infarct remodelling. Isolated mouse WT myocytes, loaded with the potentiometric dye Tetramethyl Rhodamine Methyl Ester (TMRM) and exposed to laser light generate reactive oxygen species, a potent opener of the $\mathrm{mPTP}$. The time to mPTP opening can therefore be used as an assay of drug interaction with the mPTP. Using cyclosporine as a postive control, we found that ilomastat, unlike cyclosporine, did not delay 